\title{
Mixing and Transport in Stars
}

\author{
Vittorio M. Canuto \\ NASA, Goddard Institute for Space Studies, New York, NY $10025 \&$ \\ Dept. of Applied Physics and Mathematics, Columbia University, New \\ York, NY 10027, U.S.A.
}

Transport and mixing in stars is as important as it is difficult to quantify (Zahn 1992; Schatzman 1996; Maeder 1997; Pinsonneault 1997). A first difficulty is that both transport and mixing are dynamical processes which, given the low viscosities of stellar interiors, usually means that the flow is turbulent giving rise to technical difficulties for turbulence is still an incomplete chapter though recent studies have brought about considerable progress. A second difficulty is that turbulence is not self-sustaining and unless there is a source, dynamical mixing and transport will decay in time and eventually die out. Thus, the question: what is the source of turbulence, let alone how to describe it? In the convective zone, the source is the unstable stratification but the mixing there is so strong that one does not need a sophisticated theory to describe it. Strong turbulence is easier to describe than weak turbulence and yet the latter is when the problems become interesting and our descriptive power is less reliable. For example, below the solar CZ we don't even know for sure the source of stirring, let alone how to describe it and yet, it is the region where we would like to be confident about models. The transport of $\mathrm{Li}$ is the best example of a mixing and transport that cannot be too strong or too weak (Schlattl and Weiss 1999). A third difficulty is the unstated assumption that "transport" (advection) and "mixing" (diffusion) have different origin. This need not be so. Consider a scalar $A$ described by the equation:

$$
\frac{\partial A}{\partial t}+\overline{\mathbf{u}} \cdot \nabla A=\frac{\partial}{\partial x_{i}}\left(D_{i j} \frac{\partial A}{\partial x_{j}}\right)
$$

where $D_{i j}$ is a tensorial diffusivity. Separating the symmetric (s) and antisymmetric (a) parts of $D_{i j}$, Eq. (1) becomes:

$$
\frac{\partial A}{\partial t}+\left(\overline{\mathbf{u}}+\mathbf{u}^{*}\right) \cdot \nabla A=\frac{\partial}{\partial x_{i}}\left(D_{i j}^{s} \frac{\partial A}{\partial x_{j}}\right) \quad \text { where } \quad u_{i}^{*}=-\frac{\partial}{\partial x_{j}} D_{i j}^{a} .
$$

Equations (2) exhibit two important features: 1) only the symmetric part of the diffusivity tensor contributes to diffusion (mixing) while 2) the anti-symmetric part gives rise to transport via the advective velocity $\mathbf{u}^{*}$. Thus, turbulence gives rise to both mixing by diffusion and transport by advection. It is therefore unphysical to speak of diffusion and advection as separate phenomena since they may have the same origin. Thus, even in the absence of an average mean field $\overline{\mathbf{u}}$, there is an advective velocity $\mathbf{u}^{*}$ since in all cases we have studied, the diffusivities have an antisymmetric part. A fourth difficulty is the lack of clear distinction between active and passive scalars. Active scalars (such 
as $T$ and $\mu$ ) couple to the density and ultimately to the velocity field, thus affecting turbulence. A passive scalar like $\mathrm{Li}$ does not couple to the density and it is passively transported by the existing turbulence without influencing it. Consider the following. In general, one needs five fields, $u_{i}, T, \mu, \rho, c$ to represent velocity, temperature, mean molecular weight, mass (or density) and passive scalars. Each field $\phi$ has a mean part $\bar{\phi}$ and a fluctuating component $\phi^{\prime \prime}$. The dynamic equations for the mean variables $\overline{u_{i}}, \bar{T}, \bar{\mu}, \bar{\rho}$ and $\bar{c}$ require the knowledge of the fluxes

$$
\tau_{i j}=\overline{u_{i}^{\prime \prime} u_{j}^{\prime \prime}}, \quad J_{i}^{h}=\overline{u_{i}^{\prime \prime} T^{\prime \prime}}, \quad J_{i}^{\mu}=\overline{u_{i}^{\prime \prime} \mu^{\prime \prime}}, \quad J_{i}^{\rho}=\overline{u_{i}^{\prime \prime} \rho^{\prime \prime}}, \quad J_{i}^{c}=\overline{u_{i}^{\prime \prime} c^{\prime \prime}}
$$

that represent momentum flux (Reynolds stresses), heat flux, $\mu$-flux, mass flux and concentration flux. We have several diffusivity tensors. In a 1D case, the diffusivities for the density and a passive scalar are given by (Canuto 1999):

$$
D_{\rho}=\left(D_{h}-D_{\mu} R_{\mu}\right)\left(1-R_{\mu}\right)^{-1}, \quad D_{c}=\left(D_{h}+D_{\mu} R_{\mu}\right)\left(1+R_{\mu}\right)^{-1}
$$

where $R_{\mu}=\nabla_{\mu}\left(\nabla-\nabla_{a d}\right)^{-1}$. The minus sign in $D_{\rho}$ is due to the fact that $T$ and $\mu$ act on the density $\rho$ in opposite sense. On the other hand, a passive scalar cannot be sensitive to $T$ and $\mu$ individually but only to their total contribution. Thus, $D_{\rho}$ and $D_{c}$ must be different. A few examples may help.

a) Overshooting, OV. Overshooting is a prototype example of mixing (Canuto $1999 ; 2000)$. The problem has both unsolved and unresolved aspects: the unsolved part is that we do not yet have an expression for practical calculations while the unresolved issue is that there is no agreement on which variable determines the extent of the OV. Roxburgh (1978) used the convective flux $J_{h}$ to gauge the extent of the OV. Even if one accepts $J_{h}$ as the "indicator" of the OV, the cited criterion cannot be used unless one supplements it with several ingredients that the model does not provide (Canuto 1997). A plume model (Schmitt et al. 1984) provides an expression for the OV of the form: $O V / H_{p} \sim w^{3 / 2} f^{1 / 2}$. Here, $w$ is the rms initial velocity, for example at the last point of the convective zone and $f$ is the filling factor representing the topology of the plumes. Since space is not equally divided between ascending and descending plumes, $f$ is a critical quantity quite difficult to compute. The above result cannot be used in practice since the model does not provide either $w$ or $f$. Recently, 2D simulations were employed (Ludwig et al. 1999). Due to the inverse cascade, 2D turbulence piles up kinetic energy at the largest scales and since such scales are diffusive, they contribute the most to the OV. If one uses $w$ as an indicator of the OV, one will naturally end up with large OV. This is, however, not a true physical feature but an artifact of having changed $3 \mathrm{D}$ with $2 \mathrm{D}$, a change that alters the physics of the problem (Canuto 2000). Finally, using a 3D large eddy simulation, Saikia et al. (2000) suggest the scaling $O V / H_{p}=F_{b}^{1 / 2}$ where $F_{b}$ is the flux at the bottom. However since overshooting concerns an active scalar, the heat flux may bear little relevance to the OV that must be described by a mass flux with a diffusivity $D_{\rho}$ that depends on both heat and $\mu$ diffusivities.

b) $\mathrm{Li}$. The case of $\mathrm{Li}$ corresponds to that of a passive scalar with a diffusivity $D_{c}$ and Eqs. (4) clearly show that $D_{c} \neq D_{\rho} \neq D_{h} \neq D_{\mu}$, a conclusion also arrived at using heuristic arguments (Pinsonneault 1997).

c) Semi-convection: $\nabla-\nabla_{a d}>0, \nabla_{\mu}>0, R_{\mu}>1$.

Data show that $D_{h}>D_{\mu}, \quad \mu($ flux $)<T($ flux $), \quad D_{\mu} R_{\mu}<D_{h}$. 
d) Salt-fingers: $\nabla-\nabla_{a d}<0, \nabla_{\mu}<0, R_{\mu}<1$.

Data show that $D_{\mu}>D_{h}, \quad T($ flux $)<\mu($ flux $), \quad D_{h}<D_{\mu} R_{\mu}$.

e) Angular momentum. The transport of angular momentum depends on the Reynolds stresses $\tau_{i j}$ which depend on shear and on the $T$ and $\mu$ gradients.

The above discussion shows that one needs a unified approach capable of encompassing the large variety of processes a)-e) which influence one another. Progress has been recently achieved (Canuto 1999) and new numerical solutions have become available. In the case $\nabla_{\mu}=0$ and $\nabla \overline{\mathbf{u}}=0$ (a $\mathrm{CZ}$ with radiative losses), the full model reduces to five differential equations which must be solved together with a stellar code. Kupka (2000) and Kupka and Montgomery (2000) have recently successfully found a mathematical algorithm that allows such a coupling to be computed for A stars. The model naturally predicts an overshooting region which is indeed quite well represented by a mass flux, as the discussion above indicates. To our knowledge, this is the first time that a turbulence model coupled to a stellar envelope code has predicted an overshooting regime. The next step is the application of the same general model to the solar OV and subsequently, the inclusion of a $\mu$-gradient will clarify the effect of a $\mu$-barrier on the OV. The extension of the above formalism to treat radiative, stable regions in the presence of shear instabilities is under way by the present author. The model leaves unspecified the nature of the source of turbulence in the stable region since we purposely want to separate the identification of the source of turbulence and its description. The strength of the source of turbulence is written in units of $\chi \Omega^{2}$ (where $\chi$ is the radiative conductivity) and is treated as an "efficiency variable". If for example, available data on the Li problem will require an efficiency far larger than the known types of shear instabilities (e.g., in the Eddington-Sweet-Vogt currents, Zahn 1992), one would have to conclude that other mechanisms (Schatzman 1996) are to be invoked. However, given the crudeness of the treatments thus far, it is premature to write off shear instabilities as done by some authors. The present formalism will, among other things, help answer this question on a firmer basis.

\section{References}

Canuto, V.M. 1997, ApJ, 489, L71

Canuto, V.M. 1999, ApJ, 518, L119; ibid., 524, 311

Canuto, V.M. 2000, MNRAS, 317, 985; A\&A, 357, 177

Kupka, F. 2000, ApJ, (submitted)

Kupka, F. \& Montgomery, M. 2000 (in preparation)

Ludwig, H.G., Freytag, B. \& Steffen, M. 1999, A\&A, 346, 111

Maeder, A. 1997, A\&A, 321, 134

Pinsonneault, M. 1997 ARAA, 1997, 35, 357

Roxburgh, I. 1978, A\&A, 65, 281

Saikia, E., Singh, H.P., Chan, K.L. \& Roxburgh, I. 2000, ApJ, 529, 402

Schatzman, E. 1996, J. Fluid Mech., 322, 355

Schlattl, H. \& Weiss, A. 1999, A\&A, 347, 272

Schmitt, J., Rosner, R. \& Bohn, H.U. 1984, ApJ, 282, 316

Zahn, J.-P., 1992, A\&A, 265, 115 